\title{
Multiple Component Outflows in an Active Region Observed with the EUV Imaging Spectrometer on Hinode
}

\author{
P. Bryans, ${ }^{1,2}$ P. R. Young, ${ }^{1,2} \&$ G. A. Doschek ${ }^{1}$
}

\begin{abstract}
We have used the Extreme Ultraviolet Imaging Spectrometer (EIS) on the Hinode spacecraft to observe large areas of outflow near an active region. These outflows are seen to persist for at least 6 days. The emission line profiles suggest that the outflow region is composed of multiple outflowing components, Dopplershifted with respect to each other. We have modeled this scenario by imposing a double-Gaussian fit to the line profiles. These fits represent the profile markedly better than a single Gaussian fit for Fe XII and XIII emission lines. For the fastest outflowing components, we find velocities as high as $200 \mathrm{~km} \mathrm{~s}^{-1}$. However, there remains a correlation between the fitted line velocities and widths, suggesting that the outflows are not fully resolved by the double-Gaussian fit and that the outflow may be comprised of further components.
\end{abstract}

\section{Introduction}

One of the most significant discoveries of the Extreme Ultraviolet Imaging Spectrometer (EIS) on the Hinode spacecraft is the detection of large areas of outflowing plasma at the boundaries of active regions (Doschek et al. 2007; Sakao et al. 2007; Harra et al. 2008; Doschek et al. 2008). These outflowing regions were found to occur in areas of low line emission intensity, often adjacent to coronal loops. Doschek et al. (2007) also found the outflowing regions to exhibit larger spectral line widths than found in the much brighter active region closed loops. These line widths are in excess of pure thermal Doppler broadening. Doschek et al. (2008) subsequently found a strong positive correlation between the outflow velocity (the Doppler shift of the line emission) and the non-thermal velocity (the width of the emission line). Harra et al. (2007) have related the outflowing regions to coronal mass ejections and Harra et al. (2008) and Doschek et al. (2008) have postulated that the outflowing regions could be contributors to the solar wind. This conclusion is consistent with results

\footnotetext{
${ }^{1}$ Naval Research Laboratory, 4555 Overlook Ave. SW, Washington DC 20375

${ }^{2}$ George Mason University, 4400 University Dr., Fairfax VA 22030
} 
based on completely independent studies of the heliospheric magnetic field, for example by Schrijver \& De Rosa (2003). Given such implications, the further characterization of outflowing regions could be important for the understanding of fundamental physical processes involved in production of the solar wind and mass flow into the corona.

Emission line widths in excess of their thermal Doppler widths have been observed since the rocket flight analysis of Boland et al. (1975). However, the origin of the spectral broadening remains unclear; possible explanations include turbulence in the atmosphere due to magnetic reconnection (Parker 1988) and the presence of coronal waves (Mariska 1992). Earlier studies of non-thermal line broadening did not link the broadening to bulk mass flows because there were few high resolution EUV-UV solar spectra coupled to images such that either the broadening or bulk mass flows could be related to particular coronal structures. The outflows under present discussion would have been difficult to find in earlier studies because of their appearance in areas of low intensity in coronal spectral lines, making their measurement difficult. It was not until the EIS instrument that the required spatial and spectral resolution became available to accurately determine the spectral properties of the outflowing region and marry these with magnetic structures of the solar atmosphere. Of particular interest among the EIS findings is the correlation of line shift with line width found by Doschek et al. (2008) and Hara et al. (2008). The fact that those emission lines that show the largest non-thermal Doppler velocities also display the largest widths raises the possibility that the outflows may result from multiple flow sites, all Doppler shifted relative to one another. Spectral lines at the EIS spatial and spectral resolution might be convolutions of line emission from multiple unresolved flow sites. This possibility was suggested by both Doschek et al. (2008) and Hara et al. (2008) but was not explored further; their spectral analyses assumed simple Gaussian line profiles.

An object of the present paper is to determine whether the apparent excess line widths can be attributed to the line emission being poorly represented by a single Gaussian. We expand upon the previous works dealing with outflows in EIS spectra by attempting to model the outflowing plasma as a blend of outflow sites with different flow speeds. This method of analysis depends on the different flow velocities being sufficiently shifted in relation to one another as to be spectrally resolved. We thus focus on observations that have previously been identified as displaying highly asymmetrical line profiles (Doschek et al. 2008). We also limit our present model to an outflow region that can be well-represented by a sum of two flow velocities, with the emission line profiles modeled as a sum of two Gaussian components. The remainder of this paper is organized as follows: in Section 2 we outline the observations and explain the EIS data reduction procedures. In Section 3 we present the results of the double Gaussian line fitting technique and discuss possible interpretations in Section 4 . 


\section{Observations and Data Reduction}

The EIS instrument on Hinode is described by Culhane et al. (2007); more details are also given by Korendyke et al. (2006). The Hinode mission is described by Kosugi et al. (2007). The EIS observations discussed in the present paper utilize the $1^{\prime \prime}$ slit rastered from west to east in $1^{\prime \prime}$ increments to build up a two-dimensional spectral image. The two observing programs used here raster areas of $256 \times 256$ and $460 \times 384$ pixels, returning 20 and 24 spectral windows, respectively.

For the purposes of this paper we have analyzed the active region (AR) 10978. This AR was previously identified as displaying an outflow region with possible multiple components by Doschek et al. (2008). Del Zanna (2008) measured outflows from a single active region and found that they persisted for at least 4 days. We expand on these two works by tracking the evolution of the AR observed by Doschek et al. (2008) over 7 days and characterize the outflows through two Gaussian rather than single Gaussian fits to the emission line profiles. We use 10 separate EIS raster observations spanning from 2007 December 09 to 2007 December 15. To give context of the size and location of the AR, we show full disk Extreme Ultraviolet Imaging Telescope (EIT) images at Dec 09 00:00, Dec 12 11:12, and Dec 15 18:13 in Figure 1, The EIS field of view is highlighted as orange boxes in Figure 1 and the times and locations of the EIS observations are summarized in Table 1, The locations are given in arcseconds relative to Sun center, with positive positions north and west. Exposure times are for each position in the raster. We label the observations 1-10 for ease of reference henceforth.

We have reduced the observational data using the standard EIS software data reduction package eis_prep as described in the SolarSoft software distribution (Freeland \& Handy 1998). This includes flagging any saturated data as missing, removing the CCD pedestal and dark current, removing cosmic rays and hot pixels, and accounting for detector bias. In addition, there exists a misalignment between the EIS CCD and slit that causes the observed spectra to lie at an angle to the CCD column. The magnitude of this 'slit tilt' has been estimated by averaging the velocities over a large raster of the quiet Sun and we use these values to correct for its effect here. Finally, there is an instrumental effect-due to the temperature variation over the Hinode orbit - that causes a quasi-periodic variation in the line centroid position. We corrected for this 'thermal drift' by selecting a quiet Sun region near but outside of the active region for each raster observation and assumed that the average of the line centroids in this region represented the rest wavelength. This allows an adjustment of all the wavelengths in the raster that removes the orbital drift. In some of the observations the South Atlantic Anomaly (SAA) results in enhanced particle hits on the detector. This effect occurs in a particular part of the Hinode orbit and results in vertical 
stripes of noise on the raster images. These areas have been omitted from the analysis.

The Fe XII emission line at $195.12 \AA$ is the strongest line observed in the observations analyzed here. Due to the high count statistics, this line is commonly used when deriving both thermal and non-thermal plasma velocities from EIS observations. However, care must be taken when analyzing this line due to a blend with another Fe XII line at $195.18 \AA$. The ratio of these two lines depends on electron density. In addition to resulting in an increase in the observed line width, the presence of this blend can also shift the line centroid longward of the actual wavelength of the $195.12 \AA$ emission, depending on the density. The density of the outflow region analyzed here was calculated by Doschek et al. (2008) to be $\lesssim 10^{9} \mathrm{~cm}^{-3}$. At these densities the $195.18 \AA$ line should not significantly affect the $195.12 \AA$ emission (see Young et al. 2009).

There are two other Fe XII lines at similar wavelengths that are not blended: at $192.39 \AA$ and $193.51 \AA$. These lines have emissivities of the order of $1 / 3$ and $2 / 3$ that of the $195.12 \AA$ line, respectively. Unfortunately, the position of the $193.51 \AA$ line on the detector is contaminated with dust so a section of the observed data is lost. For the observations described in this paper, this missing section happens to be directly aligned with the outflowing region so a significant portion of the data from the outflow is not available. In addition to the Fe XII lines, we analyze emission from Si viI $275.67 \AA$, Fe VIII 185.21 $\AA$, Fe x $184.54 \AA$, Fe XIII $202.04 \AA$, Fe XIV $264.79 \AA$, Fe XIV 274.20 A, Fe XV 284.16 $\AA$ and Fe XVI 262.98 $\AA$. These emission lines come from ions with formation temperatures spanning $4.0 \times 10^{5}-2.5 \times 10^{6} \mathrm{~K}$ (Bryans et al. 2009).

As explained in Section 1, an observation of this AR has previously been studied by Doschek et al. (2008, observation 4 of the present paper) and in this paper we model the line profiles as the sum of two Gaussians with a linear background. Figure 2 shows examples of the Fe XII 192.39 $\AA$ and $195.12 \AA$ and Fe XIII 202.04 $\AA$ spectra for an individual spatial pixel in the observation 4 outflow region. Each example shows significant emission shortward of the peak emission wavelength. These asymmetric blue wings are typical of the line emission in this region.

To ensure a robust fit to the line profiles we require that the two Gaussians have the same width. While this assumption is not necessarily true of the emission, we have to impose some restrictions on the fitting because leaving every parameter to vary freely results in unsatisfactory fits. Figure 2 shows examples of these fits. We subsequently refer to the largest (in intensity) Gaussian as the primary component and the smaller, blue-shifted Gaussian as the secondary component. 


\section{Results}

A double-Gaussian fit was performed over each observation region for each of the observed emission lines. Figure 3 shows the intensity maps (from the sum of both Gaussians) of the Fe XII $195.12 \AA$ line for each observation. The emission from the primary component is dominant, and significant contribution from the secondary component is only seen in isolated regions, as shown in Figure 4, We show red contours of the secondary component on Figure 3 to indicate the location of the outflows.

Figure 5 shows the line centroid shift of the primary component of the Fe XII $195.12 \AA$ line for each raster. Comparison of Figures 4 and 5 show that the outflowing regions are also those that display a secondary Gaussian component in their emission profiles. It is also interesting to note the evolution of the outflowing region over time. We initially observe (9 December) outflowing material directly to the west of the AR and subsequently observe outflowing regions on both the east and west of the AR. While the western outflow region remains for the entire observing period, the apparent velocity of the flow is significantly decreased by the final observation (15 December). While this may suggest that the region of plasma outflow is changing position with time, it is probable that this is a line-of sight effect due to the solar rotation. The AR loop system in the early observations is oriented close to the east limb which would tend to obscure low-lying outflowing plasma to the east of the active region (trailing component). As the Sun rotates, the loops then obscure the western portion (leading component) of the outflow as the active region approaches the west limb. We discuss these projection effects further in Section 4. What is clear, however, is that the outflow persists for the entire duration that the AR is observable.

As an aside, a brief discussion of the apparent red-shift of the AR loops is warranted. While Figure 5 appears to suggest downflowing plasma at the core of the AR, this red-shift is actually due to a blend in the Fe XII $195.12 \AA$ line. We previously noted, in Section 2 , that this blend is insignificant at the low density of the outflowing region. However, the higher density of the core of the AR is sufficient to increase the relative intensity of the Fe XII 195.18 $\AA$ line such that the perceived centroid and width of the Fe XII $195.12 \AA$ line

are altered. A full discussion of this blend can be found in Young et al. (2009), but we note here that the red-shift of the AR core is a density effect rather than plasma flow.

Comparison of Figures 4 and 5 shows some interesting differences between the western and eastern outflow regions. The secondary component of the western region, while strongest at the base of the outflow region, is seen to be present almost entirely throughout the outflowing region for observations 1 through 8 . However, the same is not true of the eastern region. This is best illustrated in observations 9 and 10 where one can see extended areas of outflow in Figure 5, but only isolated regions of intensity of the secondary Gaussian 
component in Figure 4 .

In Figure 6 we show histograms of the flow velocity of the primary Gaussian component at each observation time. The velocity is calculated as the shift in line centroid from a reference wavelength of $195.12 \AA$, assumed to be the rest wavelength in constructing Figure 5 . For each plot in Figure 6 (representing a different observation) we show velocity histograms for the western outflow region as black lines and those for the eastern outflow region as red lines. We have isolated the outflowing regions from the surrounding plasma by including in these histograms only those pixels where the intensity of the secondary Gaussian is at least $10 \%$ of the primary Gaussian. Over the 10 rasters, we find a median velocity in the range $\sim 5-13 \mathrm{~km} \mathrm{~s}^{-1}$ for the western outflow region, and $\sim 0-10 \mathrm{~km} \mathrm{~s}^{-1}$ for the eastern outflow region. For the western outflow region of observation 4, Doschek et al. (2008) reported maximum flow speeds on the order of $45 \mathrm{~km} \mathrm{~s}^{-1}$ with a median shift of $\sim 20 \mathrm{~km} \mathrm{~s}^{-1}$. Our results for the flow speed of the primary Gaussian are slower than the Doschek et al. (2008) values, as one would expect from fitting a double Gaussian rather than a single to the emission profile. However, it is interesting to note that the apparent outflow observed from a single Gaussian fit cannot be entirely explained by the presence of the secondary Gaussian component; we find that the primary Gaussian is also shifted shortward of the rest wavelength.

The more interesting result is the velocity derived from the secondary component. Histograms of the same format as Figure 6 are plotted for the secondary Gaussian in Figure 7. Here we find extremely large velocities: a median of $\sim 100-130 \mathrm{~km} \mathrm{~s}^{-1}$ for the western outflow region and $\sim 90-120 \mathrm{~km} \mathrm{~s}^{-1}$ for the eastern outflow region. For both regions, some spatial pixels show velocities as high as $200 \mathrm{~km} \mathrm{~s}^{-1}$.

As outlined in Section 1, previous works have found a correlation between the outflow speed and line width in AR outflows. We can determine whether our new double Gaussian fitting resolves this correlation by plotting the Doppler velocity versus the full width at half maximum (FWHM) of both Gaussian components. In Figures 8 and 9 we show these relations for the primary and secondary Gaussian components respectively. We use black dots to represent the western outflow region and red dots to represent the eastern outflow region. Fitting two Gaussians to the spectrum rather than one naturally reduces the width with respect to a single Gaussian fit. However, there remains some evidence of a correlation between the velocity and width in the primary component. This may suggest that the double Gaussian fit still does not resolve the outflow region; we discuss this further in Section 4 .

The results discussed thus far have pertained to the Fe XII $195.12 \AA$ emission. For the other Fe XII lines, at $192.39 \AA$ and $193.51 \AA$, we find results that match well with those of the Fe XII 195.12 $\AA$ emission described above. Results from Fe XIII 202.04 $\AA$ are also in 
accordance with those of the Fe XII lines. Low temperature lines from Si VII and Fe VIII were not found to have any significant asymmetry to the emission profile and could be accurately represented by a single Gaussian without the need for a secondary blue-shifted component.

The greatest difficulty in analyzing emission from ions spanning a greater temperature range is finding lines that are free of blends. The Fe $\mathrm{x}$ emission line at $184.54 \AA$ is close to another line at a slightly shorter wavelength. Contamination from this line does not allow a satisfactory double Gaussian to be fit to the $184.54 \AA$ emission. Similarly, emission from Fe XIV $274.20 \AA$ is blended with a Si VII line which hinders a double Gaussian fitting.

At higher temperatures, emission from Fe XIV $264.79 \AA$, Fe XV $284.16 \AA$ and Fe XVI $262.98 \AA$ are not affected by nearby lines. However, while emission from these ions is strong in the core of the active region, emission in the outflowing regions is relatively weak. It is thus difficult to satisfactorily fit a double Gaussian profile to the emission. It is possible to improve the count statistics of these emission lines by summing adjacent pixels. We have done this for areas of $5 \times 5$ pixels in size, increasing the detected flux by a factor of 25 at the expense of spatial resolution. For these lines we again find no significant asymmetry to the emission profile. We do, however, find a single Gaussian fit to display outflow velocities of the same order as those determined from the primary component of the double Gaussian fits to the Fe XII and Fe XIII lines.

\section{Discussion and Conclusions}

As shown in Figure 2, a double Gaussian representation of the emission line profiles is a more accurate fit to the data than a single Gaussian representation for certain emission lines in the outflowing region. From the results presented in Section 3, we see that the double Gaussian representation is only applicable to the outflowing regions. Within these regions we find that the outflow is not only due to the fast moving secondary Gaussian component, but the primary component is also blue-shifted (see Figure 5).

By its nature the double Gaussian fit results in a reduction in the Doppler velocity of the primary component compared to that of a single Gaussian fit. We find velocities for this

component of the order of $10 \mathrm{~km} \mathrm{~s}^{-1}$, compared to $\sim 20 \mathrm{~km} \mathrm{~s}^{-1}$ found by Doschek et al. (2008) for the same outflow. However, the secondary component exhibits significantly greater outflow velocities, often as large as $200 \mathrm{~km} \mathrm{~s}^{-1}$.

Outflows are found to persist for the 6 day duration of the observations. In Figure 10, we show the variations in the derived flow velocities over this period. Here, we have compared the velocities of the primary and secondary flow components in both the eastern and western 
outflowing regions, and also indicated the standard deviation of velocities throughout the respective regions. Despite the observations spanning a significant extent of the solar rotation, there is relatively little change in the line-of-sight velocities over this time. The western outflow region (black lines in Figure 10) show a slight decreasing trend with time (for both the primary and secondary components). The eastern outflow region (red lines) shows a more pronounced, increasing velocity, trend over time. From the geometry of the AR, these apparent flow speeds are what we would expect, although we do note that there is little statistical significance to the trends. It seems evident that the outflow is not confined to a narrow cross-sectional area but, rather, emanates over a wide 'cone' of emission. The large standard deviation in the velocities shown in Figure 10 further supports this interpretation. As discussed in Section 3, the outflowing regions are also obscured by the overlying AR loop system, which further complicates the analysis.

While the double Gaussian fit appears to represent the Fe XII $195.12 \AA$ spectra well, the results displayed in Figure 8 suggest that the double Gaussian does not describe the outflow completely. In all but the first two observations there is a correlation between the primary line velocity and width in at least one of the two outflowing regions. If the assumption that the excess line width is due to multiple flow components then this remaining correlation suggests that a double Gaussian fit does not resolve the flow components. The EIS rasters for observations 1 and 2 do not cover the entire outflowing region, Figures 3 and 4 show that the outflow region is to the north-west of the raster and very likely extends beyond the raster boundary. Given that we then do not have data for the entire outflowing region, it may not be surprising that the same correlation between velocity and width is not found for these two observations.

This correlation that is seen in the primary Gaussian component is not seen for the secondary component (see Figure 9). One should be careful on the conclusions drawn from the width in a quantitative sense since the width of the primary and secondary components were set as equal in the fitting algorithm. However, the fact that there is no correlation between the velocity and width of the secondary Gaussian component suggests that if the outflow is indeed comprised of more than two components then the extra, unresolved, flow components are to be found within the primary component. The only exception to this is observation 5 where we see a complex relation between velocity and width, suggesting that we are not fully resolving the spectral data.

Observations 5, 6, 7, and 8 all show some evidence of regions with high velocities but low FWHM for the secondary component. The preponderance of these pixels are found in areas where the primary outflow speed is relatively low, away from the loop footpoints. This effect could be due to the direction of the outflowing material, with the primary and 
secondary components diverging on moving away from the footpoints. However, this remains speculation without knowing the precise topology of the outflowing plasma.

Using a different analysis technique on a different AR, de Pontieu et al. (2009) have also measured upflows. In contrast to our results, they found upflows throughout the AR. While our analysis technique will most readily identify a secondary component that is not excessively weaker than the primary, we do not believe we have missed the detection of a secondary component in the AR core. The primary intensity is a factor $\sim 10$ larger in the brightest areas of the $\mathrm{AR}$ core than in the region where we observe the outflow. If the secondary component had the same intensity in the AR as found in the outflowing region, our fitting technique would find a secondary component of this magnitude should it exist. However, we see no indication of a secondary component. Further, the primary component in the AR does not show the same blue-shift $\left(\sim 10 \mathrm{~km} \mathrm{~s}^{-1}\right)$ as the primary component in the outflowing region. Should a secondary component be present in the AR with similar velocity to that found in the outflowing region it would be easier to distinguish it from the primary given the larger difference in velocities of the two components. In addition, a single Gaussian fit to the emission in the outflowing region results in excess line widths in comparison to areas of the observation that show no outflow. The AR does not exhibit such excess line widths.

Finally, we note that a double Gaussian fit is a better representation than a single Gaussian only for the emission from the Fe XII and XIII lines. Emission from other ions is either too closely affected by nearby (in wavelength) emission lines or too weak in intensity to determine whether a double Gaussian fit is accurate.

The presence of high velocity outflowing plasma of a duration of several days leads to the question of where this material is deposited. It does not appear to be confined to the loop arcades of the immediate AR; rather it is seen to move along either open or highly extended field lines. This indicates significant mass flow into the corona and, in the case of open field lines, a possible contributor to the solar wind mass flow.

The velocity that we find for the slow Gaussian component compares well with models of the slow solar wind by Wang (1994) and Wang et al. (2009). These authors determined the outflow velocity from the slow solar wind originating from small coronal holes in the vicinity of active regions - closely matching the conditions of the observations analyzed in the present paper. They predicted source region outflow velocities of $11.3 \mathrm{~km} \mathrm{~s}^{-1}$ (Wang 1994) and $6.0 \mathrm{~km} \mathrm{~s}^{-1}$ (Wang et al. 2009). This is in good agreement with our results of $\sim 10 \mathrm{~km} \mathrm{~s}^{-1}$ for the slow moving component of the outflow. Comparison of in situ measurements of the solar wind are difficult because of large coronal holes both proceeding and preceding the active region in question. It is more likely that any detected solar wind originates in these 
coronal holes rather than the outflowing regions analyzed here.

At the 1 arcsec spatial scale of the EIS observations discussed in this paper, we are unable to spatially resolve the separate components of the outflowing material. Thus, observations of a resolution significantly better than the $1^{\prime \prime}$ level will be required for future instrumentation in order to resolve these structures. Also, given the temperature dependence of these fast outflows, spectroscopic information on such a spatial scale is needed to fully determine the nature of such phenomena.

We thank I. Ugarte Urra and Y.-M. Wang for helpful discussions and suggestions.

Hinode is a Japanese mission developed and launched by ISAS/JAXA, collaborating with NAOJ as domestic partner, and NASA (USA) and STFC (UK) as international partners. Scientific operation of the Hinode mission is conducted by the Hinode science team organized at ISAS/JAXA. This team mainly consists of scientists from institutes in the partner countries. Support for the postlaunch operation is provided by JAXA and NAOJ, STFC, NASA, ESA (European Space Agency), and NSC (Norway). We are grateful to the Hinode team for all their efforts in the design, build, and operation of the mission.

P. B., G. A. D., and P. R. Y. acknowledge support from the NASA Hinode program.

\section{REFERENCES}

Boland, B. C., et al. 1975, MNRAS, 171, 697

Bryans, P., Landi, E., \& Savin, D. W. 2009, ApJ, 691, 1540

Culhane, J., L., et al. 2007, Sol. Phys., 243, 19

Del Zanna, G. 2008, A\&A, 481, L49

de Pontieu, B., McIntosh, S. W., Hansteen, V. H., \& Schrijver, C. J. 2009, ApJ, 701, L1

Doschek, G. A., Mariska, J. T., Warren, H. P., Brown, C. M., Culhane, J. L., Hara, H., Watanabe, T., Young, P. R., \& Mason, H. E. 2007, ApJ, 667, L109

Doschek, G. A., Warren, H. P., Mariska, J. T., Muglach, K., Culhane, J. L., Hara, H., \& Watanabe, T. 2008, ApJ, 686, 1362

Freeland, S. L., \& Handy, B. N. 1998, Sol. Phys., 162, 291 
Harra, L. K., Hara, H., Imada, S., Young, P. R., Williams, D. R., Sterling, A. C., Korendyke, C., \& Attrill, G. D. R. 2007, PASJ, 59, S801

Hara, H., Watanabe, T., Harra, L. K., Culhane, J. L., Young, P. R., Mariska, J. T., \& Doschek, G. A. 2008, ApJ, 678, L67

Harra, L. K., Sakao, T., Mandrini, C. H., Hara, H., Imada, S., Young, P. R., van DrielGesztelyi, L., \& Baker, D. 2008, ApJ, 676, L147

Korendyke, C. M., et al. 2006, Appl. Opt., 45, 8674

Kosugi, T., et al. 2007, Sol., Phys., 243, 3

Mariska, J., T. 1992, in The Solar Transition Region (Cambridge: Cambridge Univ. Press)

Parker, E. N. 1988, ApJ, 330, 474

Sakao, T. et al. 2007, Science, 318, 1585

Schrijver, C. J., \& De Rosa, M. L. 2003, Sol. Phys., 212, 165

Wang, Y.-M. 1994, ApJ, 437, L67

Wang, Y.-M., Ko, Y.-K., \& Grappin, R. 2009, ApJ, 691, 760

Young, P. R., Watanabe, T., Hara, H., \& Mariska, J. T. 2009, A\&A, 495, 587 

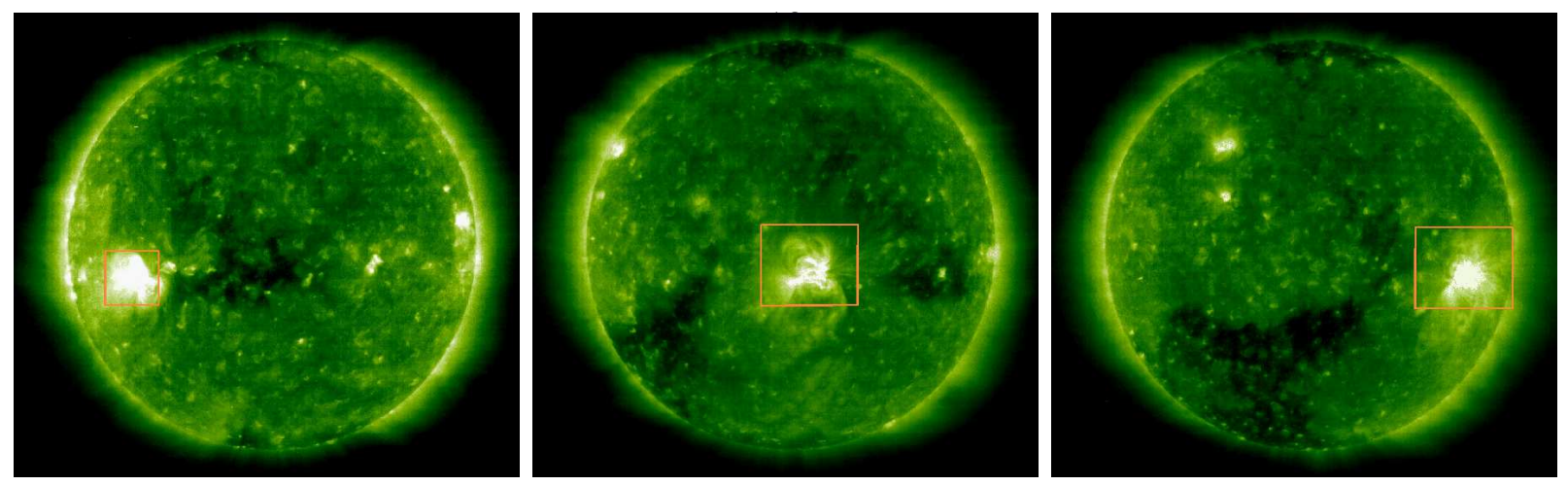

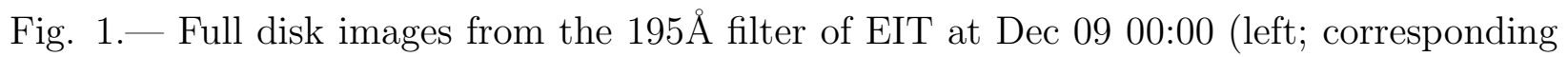
to EIS observation 1), Dec 12 11:12 (middle; corresponding to EIS observation 7), and Dec 15 18:13 (right; corresponding to EIS observation 10). Images courtesy of the SOHO EIT Consortium; SOHO is a joint ESA-NASA program.

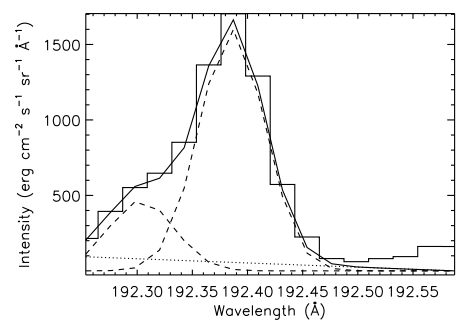

(a) Fe XII $192.39 \AA$

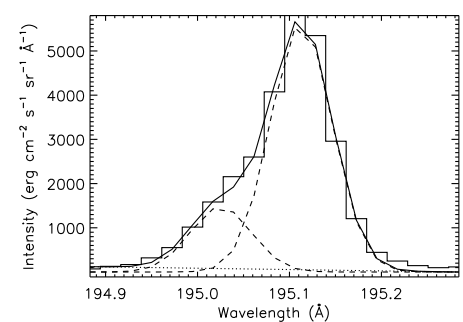

(b) Fe XII $195.12 \AA$

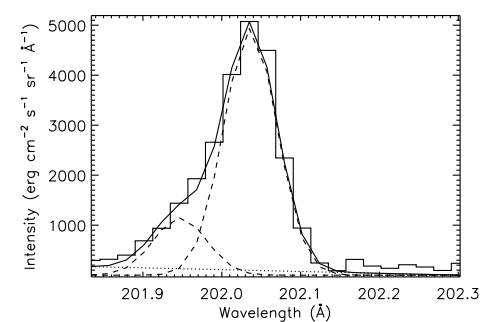

(c) Fe XIII $202.04 \AA$

Fig. 2.- Observed EIS spectra from a single pixel of the outflow region after data calibration. The chosen pixel is, in arcsec from Sun center, at $(-350,-110)$ from the observation on 2007 Dec 10 00::19:27. The double Gaussian fit is superimposed (solid curve) with the primary and secondary Gaussian components shown (dashed curves). 


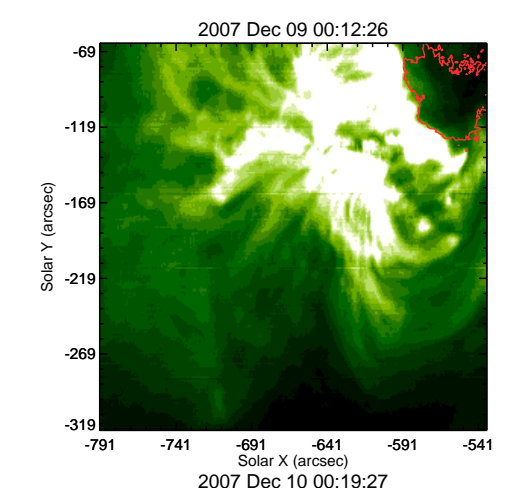

$-13-$
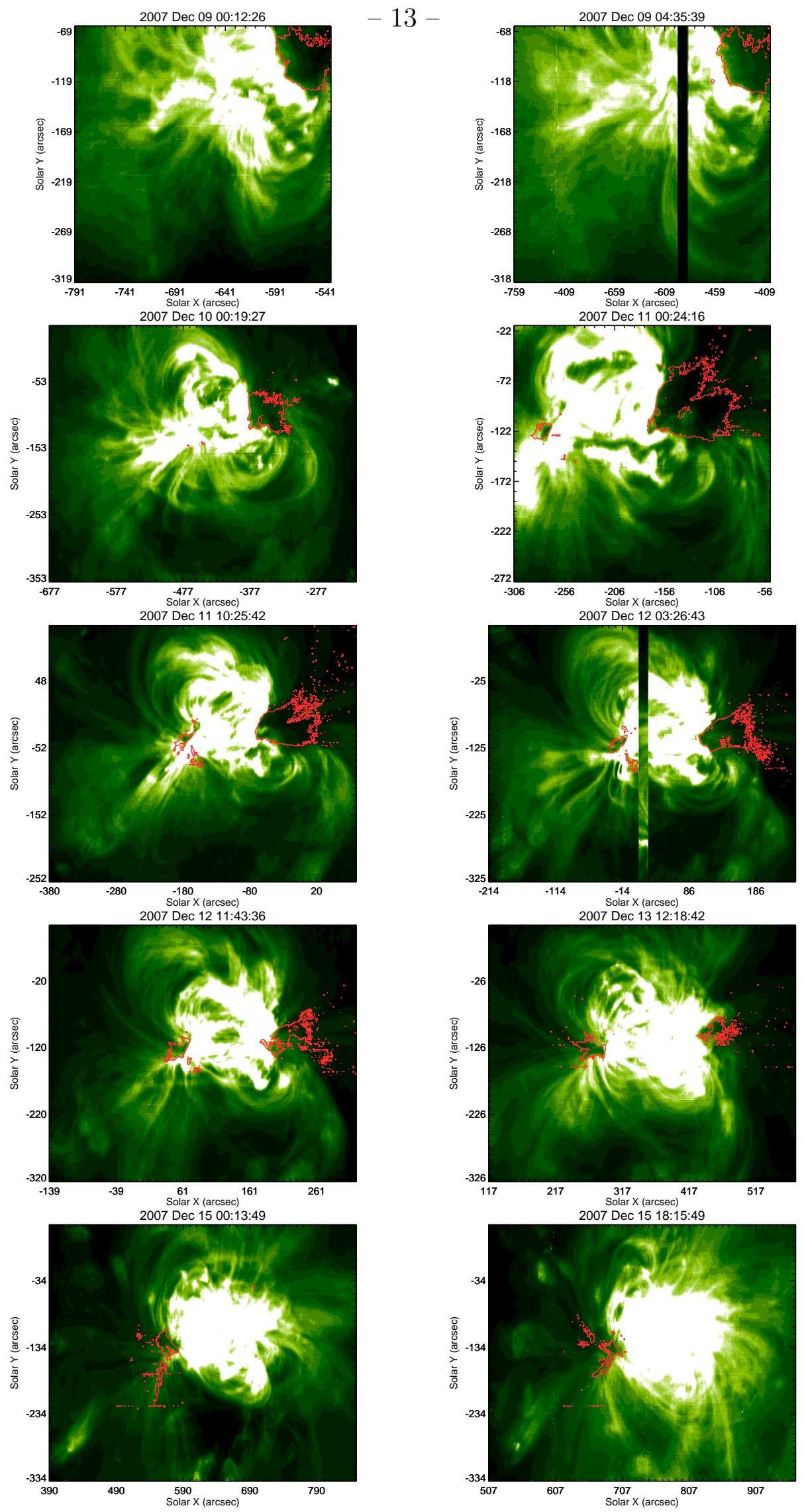

Fig. 3.- Intensity images for each of the 10 EIS observations. Time runs from left to right and top to bottom. The intensities are the sum of the intensities of the two Gaussians. The red contours show where the intensity of the secondary component is $5 \%$ of the primary. 


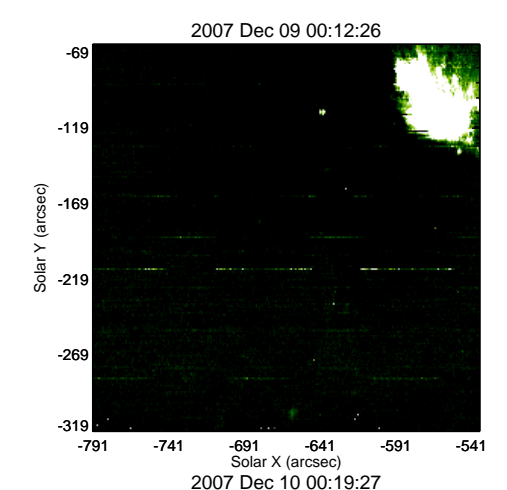

$-14-$
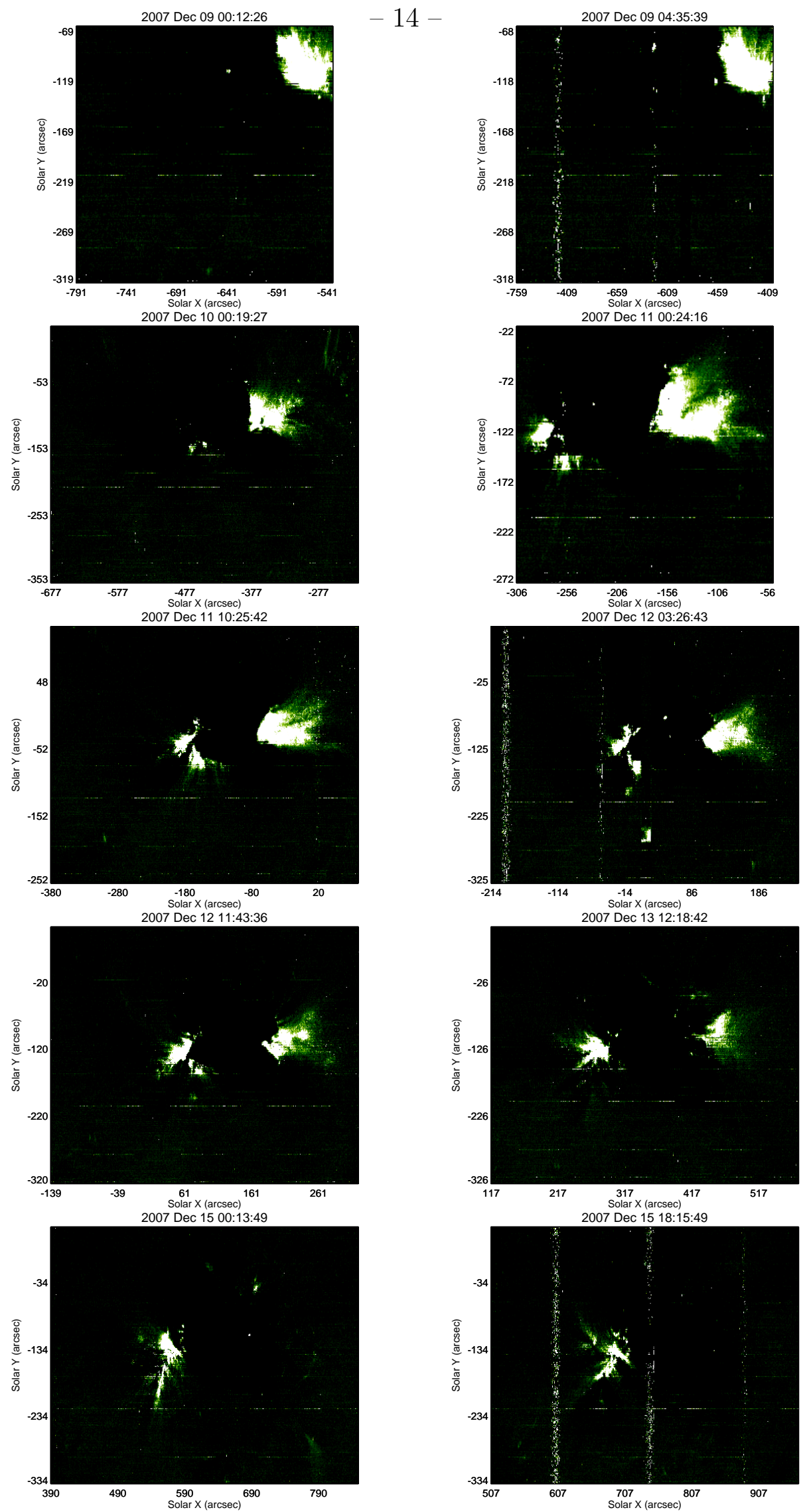

Fig. 4.- Same as Figure 3 but only showing the intensity of the secondary Gaussian. 

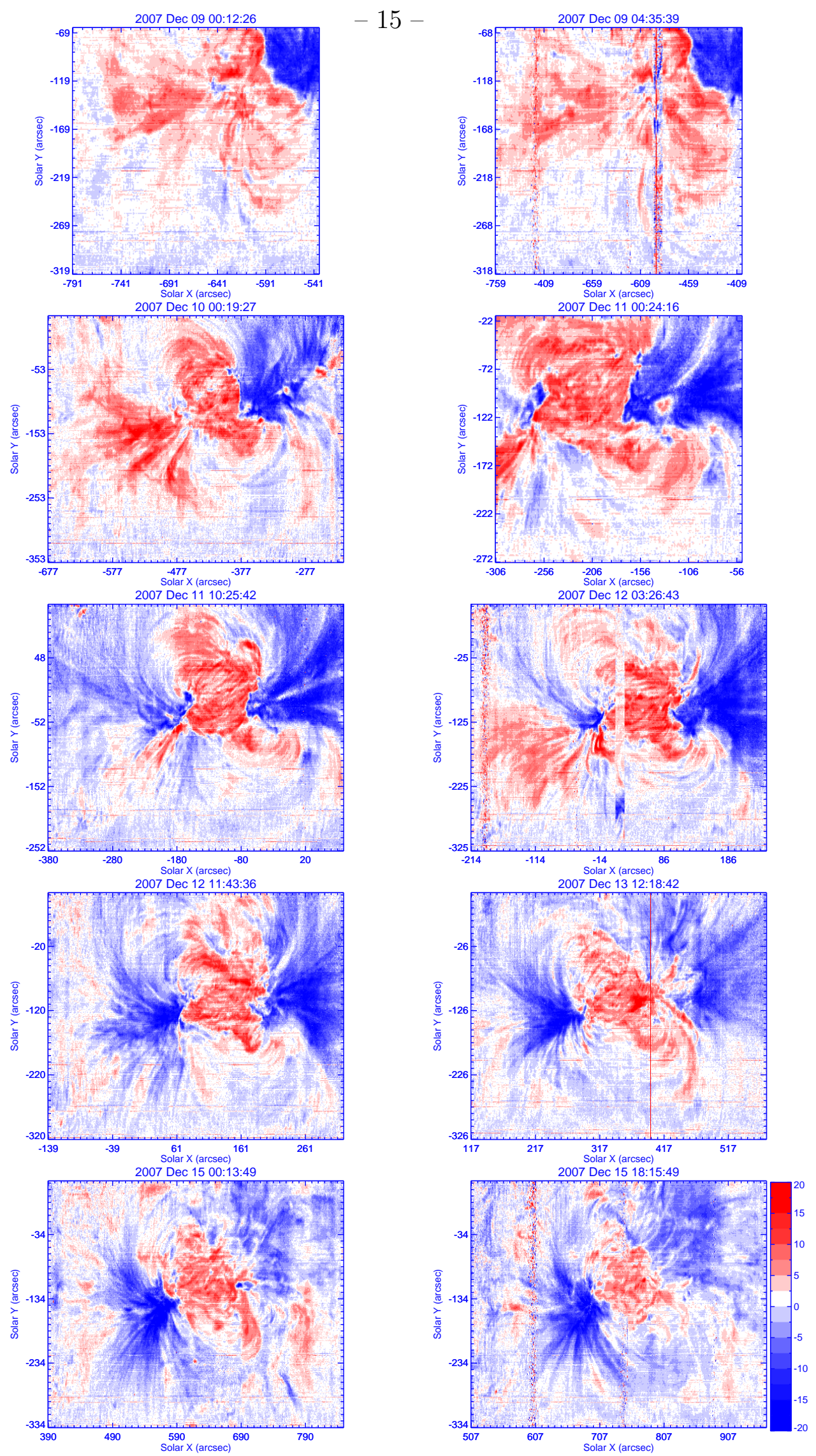

Fig. 5.- Velocity maps of the primary Gaussian for each of the 10 EIS observations. Blue represents an upflow and red a downflow. 


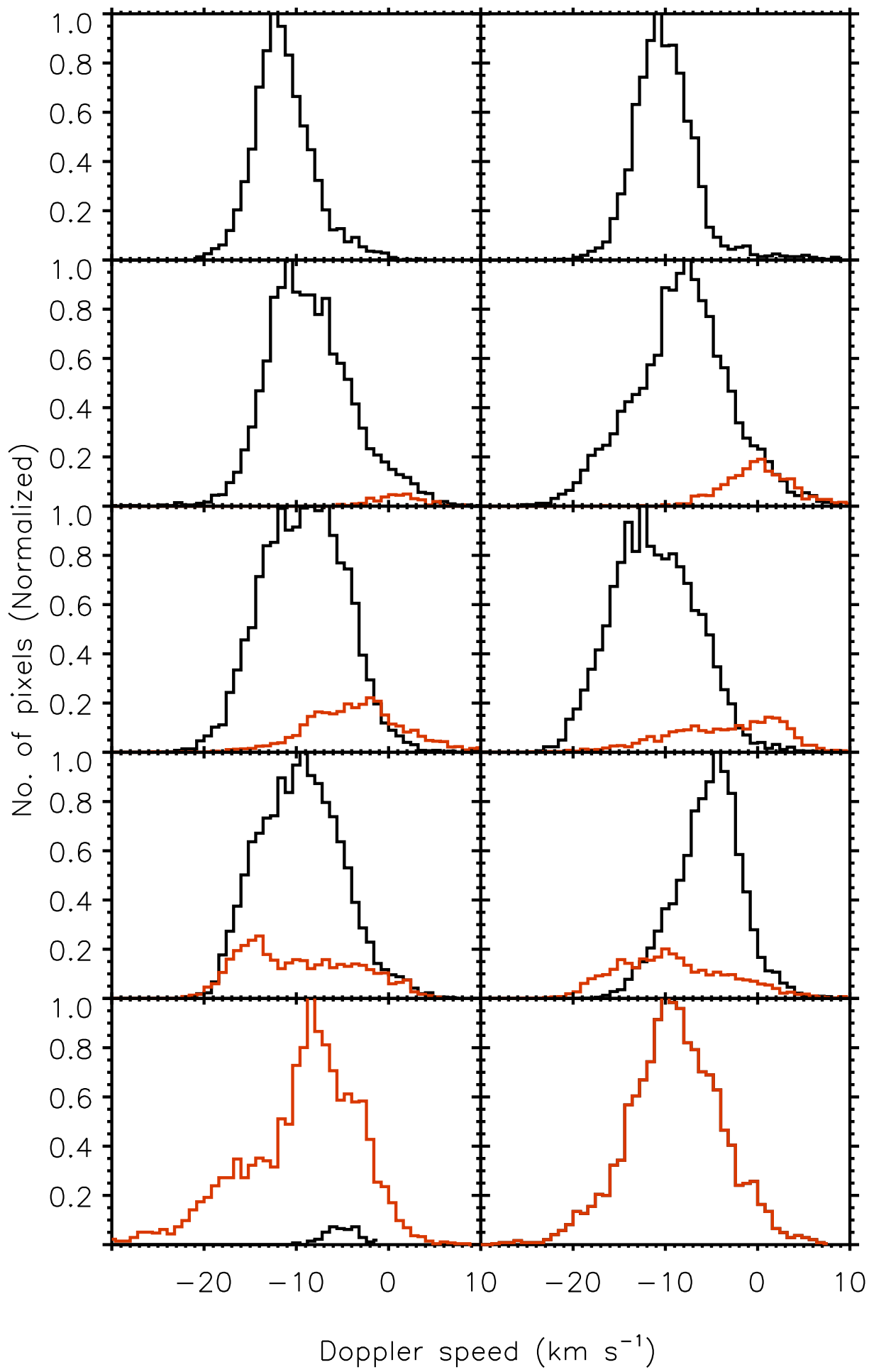

Fig. 6.- Histograms of the velocities of the primary Gaussian for each of the 10 EIS observations. Black lines represent the western outflow region and red lines represent the eastern outflow region. Only pixels where the intensity of the secondary component is at least $10 \%$ of the intensity of the primary component are plotted. The plots follow the same temporal order as Figure 3; left to right and top to bottom. 


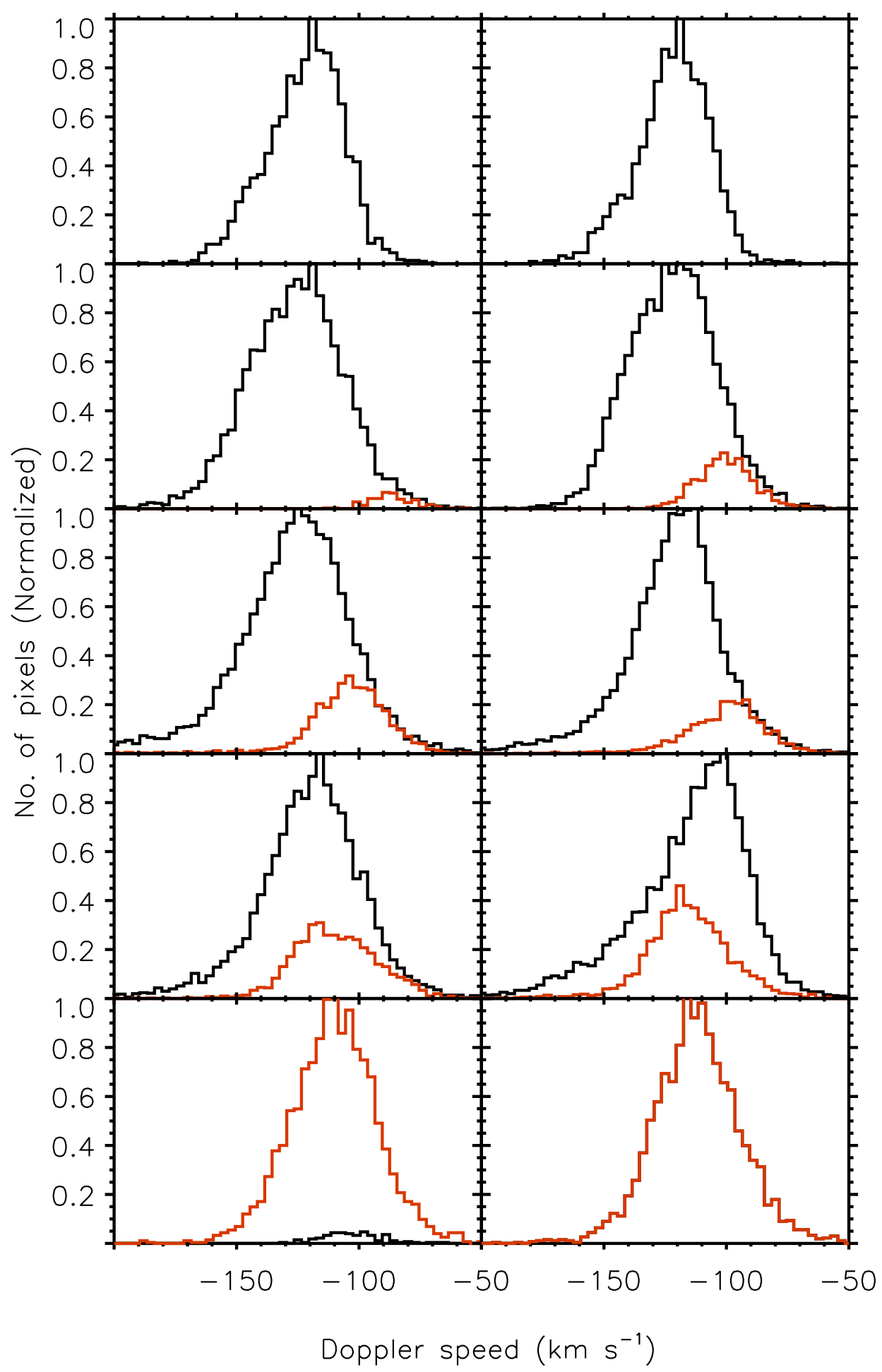

Fig. 7.- Same as Figure 6 but for the secondary Gaussian component. 


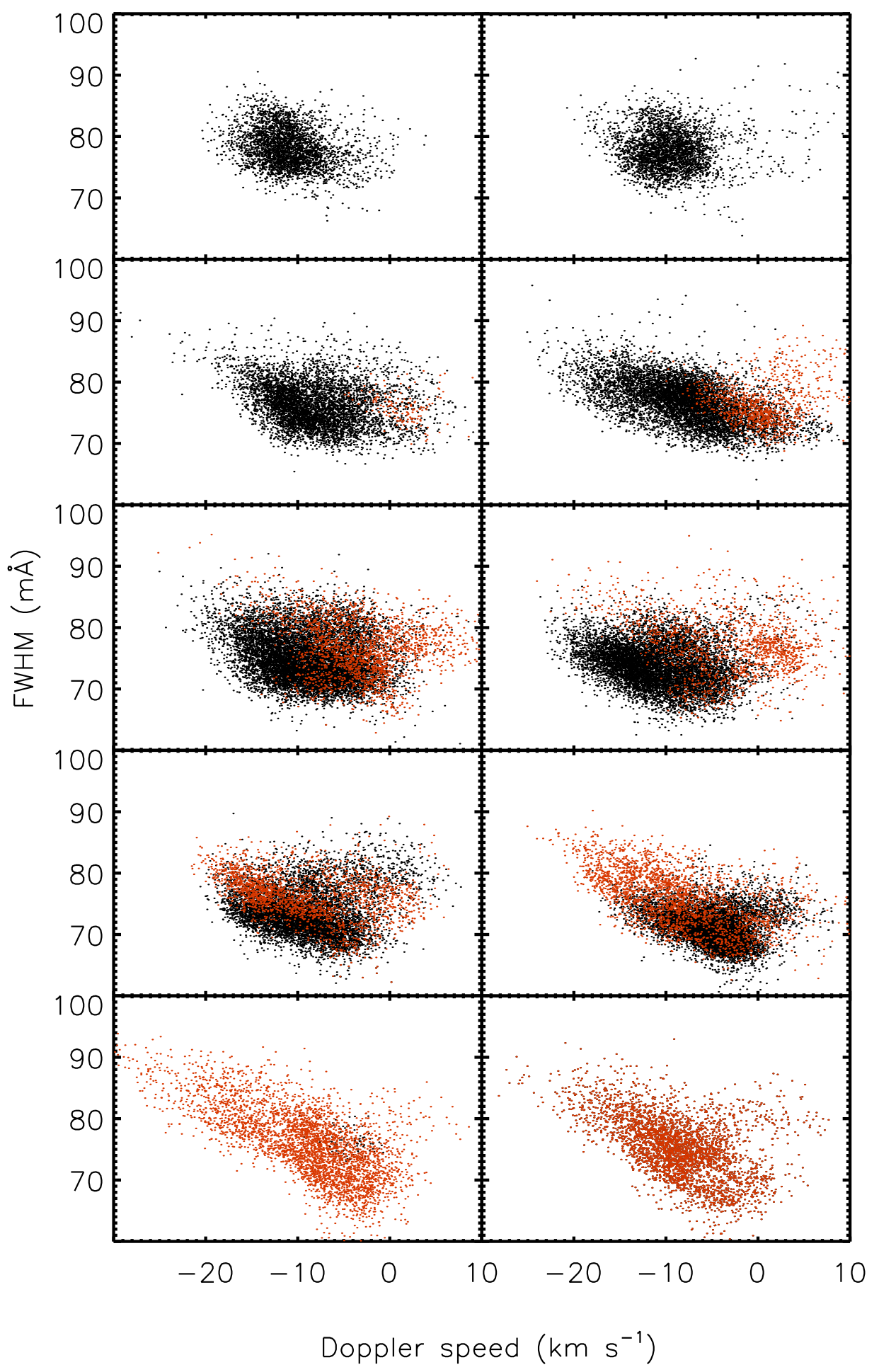

Fig. 8. - Plots of velocity (centroid shift) versus non-thermal velocity (full width half maximum) for the primary Gaussian of each of the 10 EIS observations. Black dots represent the western outflow region and red dots represent the eastern outflow region. Only pixels where the intensity of the secondary component is at least $10 \%$ of the intensity of the primary component are plotted. The plots follow the same temporal order as Figure 3, left to right and top to bottom. 


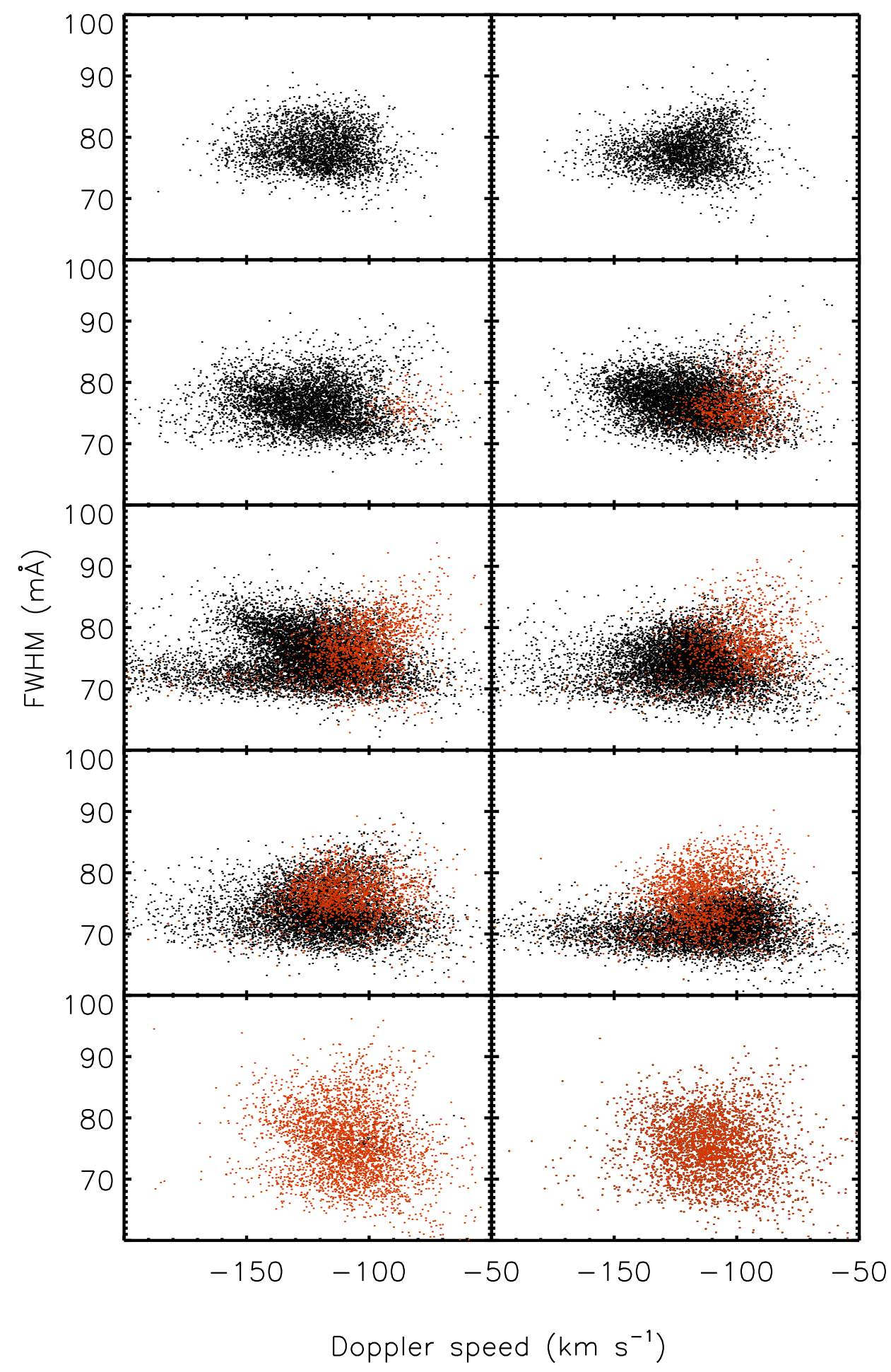

Fig. 9.- Same as Figure 8 but for the secondary Gaussian component. 

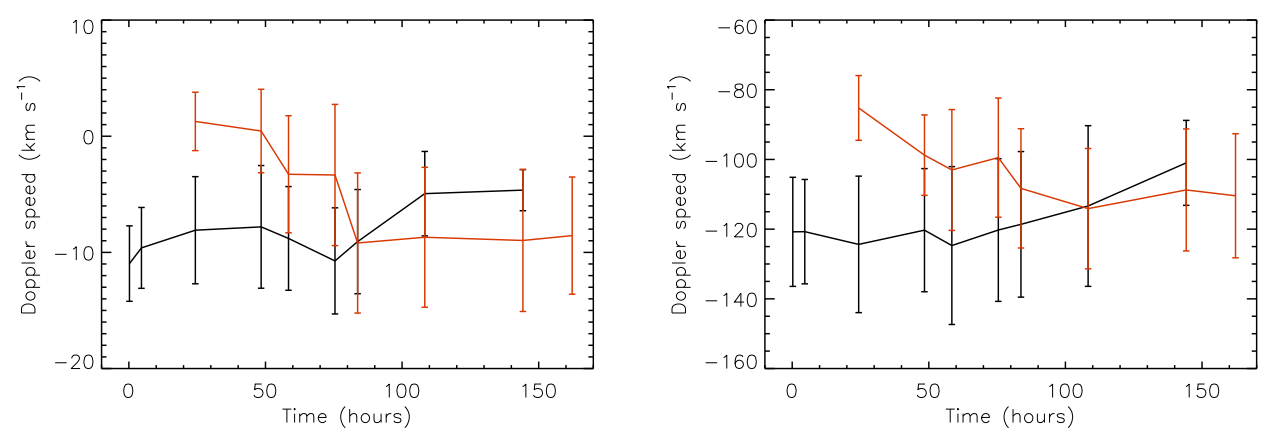

Fig. 10. - Plots of average velocity with standard deviation versus time over the duration of the EIS observations. Black lines represents average velocities from the western outflow region and red lines indicate those from the eastern outflow region. The left panel represents the primary Gaussian and the right represents the secondary Gaussian. Time is given in hours from 2007 Dec 09 00:00:00 UT.

Table 1. AR10978 Observations in 2007

\begin{tabular}{ccccc}
\hline \hline Observation Number & Date & Time (UT) & Location (arcsec) & EIS Exposure Time $(\mathrm{s})$ \\
\hline 1 & Dec 09 & $00: 12: 26$ & $-663,-191$ & 60 \\
2 & Dec 09 & $04: 35: 39$ & $-631,-190$ & 60 \\
3 & Dec 10 & $00: 19: 27$ & $-447,-161$ & 40 \\
4 & Dec 11 & $00: 24: 16$ & $-178,-144$ & 60 \\
5 & Dec 11 & $10: 25: 42$ & $-150,-141$ & 40 \\
6 & Dec 12 & $03: 26: 43$ & $16,-133$ & 40 \\
7 & Dec 12 & $11: 43: 36$ & $91,-128$ & 40 \\
8 & Dec 13 & $12: 18: 42$ & $347,-134$ & 40 \\
9 & Dec 15 & $00: 13: 49$ & $620,-142$ & 40 \\
10 & Dec 15 & $18: 15: 49$ & $737,-142$ & 40 \\
\hline
\end{tabular}

Note. - Times given are the start times of each observation. Locations are the center of the raster. Exposure times are for each position in the raster. 


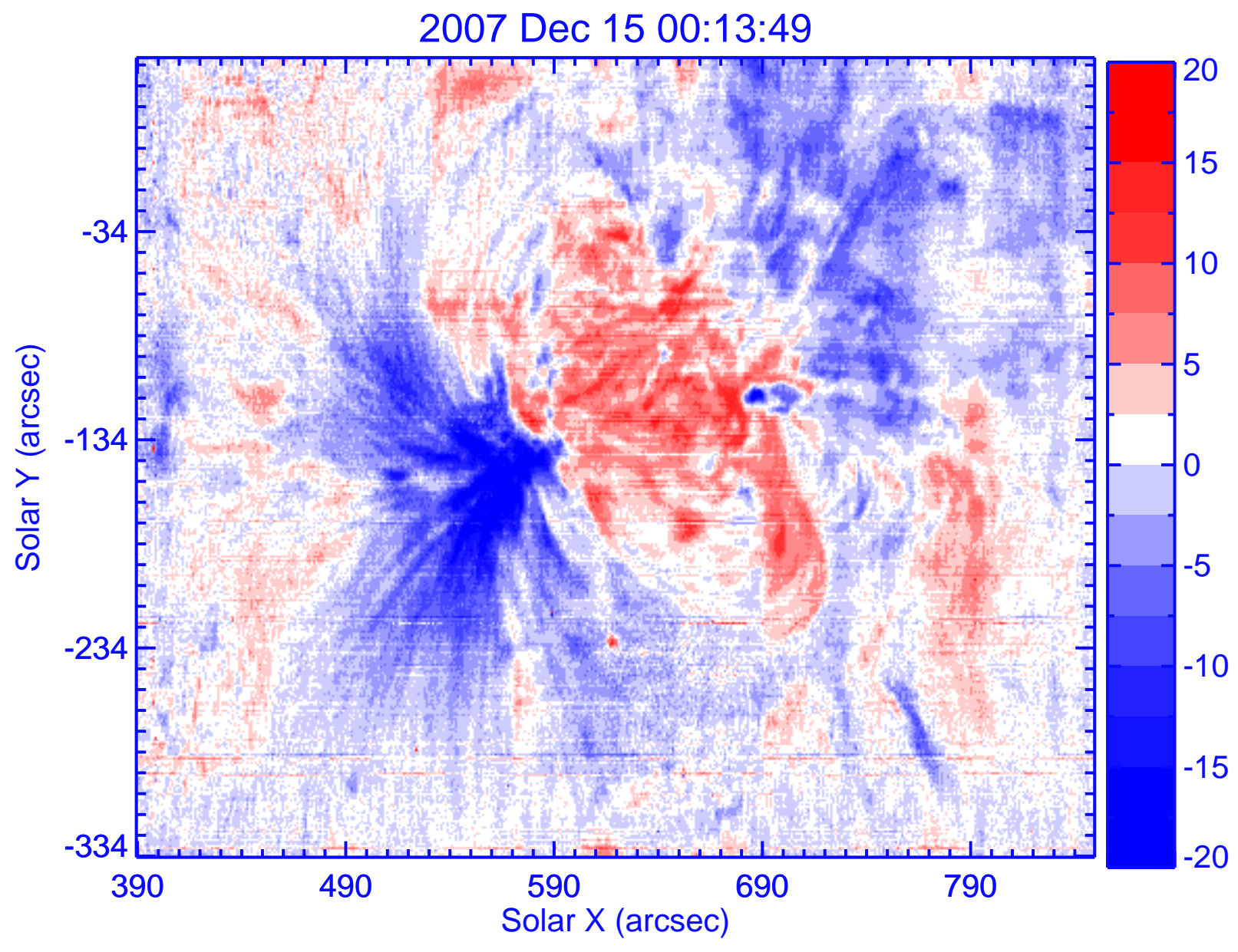




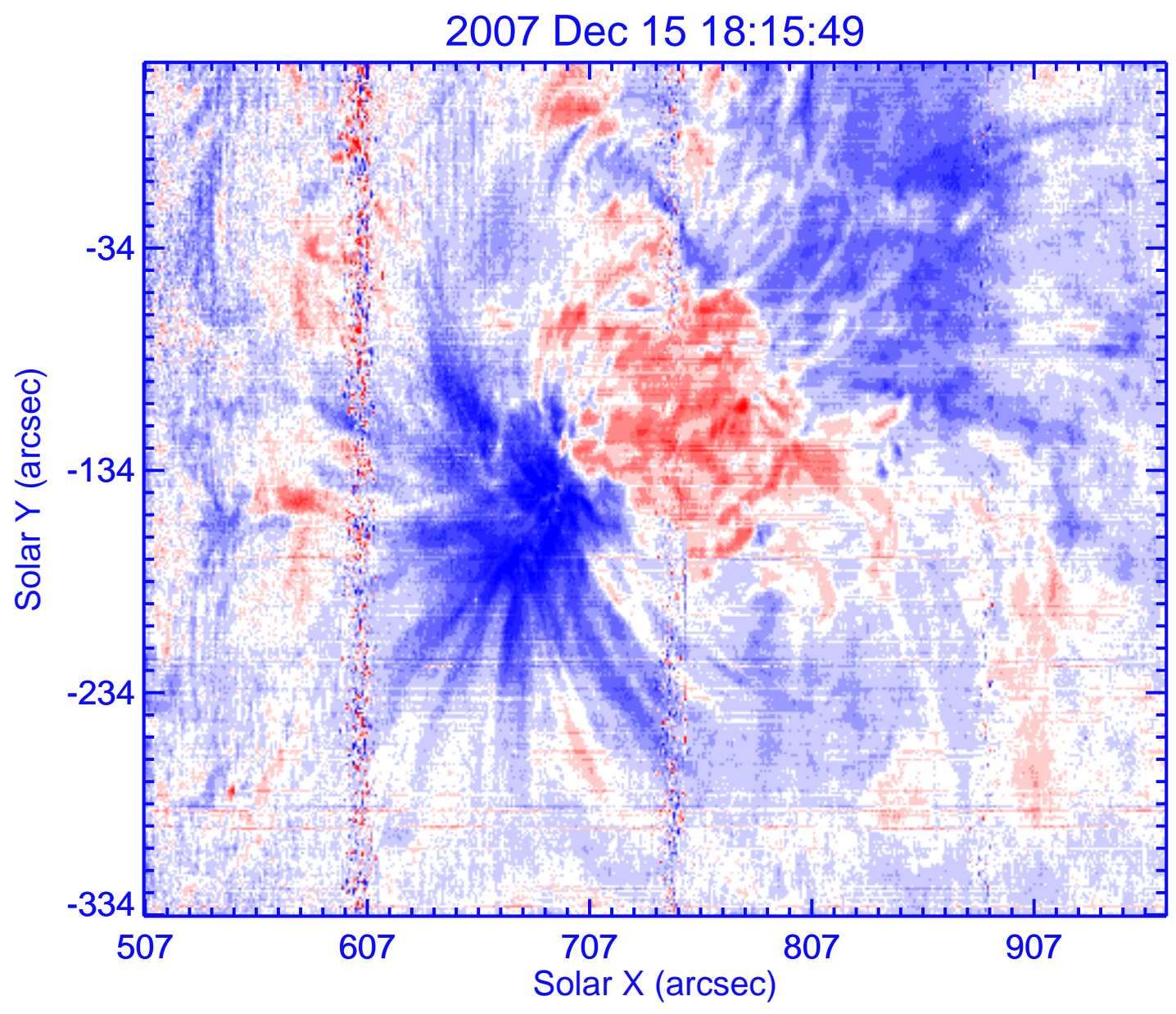

